\title{
Age-Related Changes in Rat Hippocampal Theta Rhythms: A Difference between Type 1 and Type 2 Theta
}

\author{
Yasuyuki ABE and Keiichiro TOYOSAWA ${ }^{1)}$ \\ Department of Intelligent Science, Graduate School of Science and Technology, and "Laboratory of Applied Physiology, Faculty of \\ Agriculture, Kobe University, Rokkodai, Nada-ku, Kobe 657-0013, Japan
}

(Received 14 October 1998/Accepted 6 January 1999) ABSTRACT. The age-related changes in two types of theta rhythms recorded from the hippocampus in young ( 4 months-old), mature (12-13
months-old) and aged ( $22-25$ months-old) rats were investigated. The type 1 theta rhythm was measured from hippocampal EEG recorded
from walking rats and the type 2 theta was measured from the EEG induced by reticular pontin oralis nucleus (PON) stimulation in
urethane anesthetized rats. The peak frequency and the peak power were detected from power spectra calculated on each theta sample by
fast Fourier transformation (FFT). No age-related alteration was observed on the peak frequency of type 1 theta rhythm. However, on
type 2 theta rhythm, the peak frequency was decreased in the aged rats compared with the young and the mature rats. The type 2 theta
rhythm is cholinergic, and therefore this result suggests that age-related deterioration can be clearly observed in the cholinergic system
including the hippocampus in rats.-KEY wORDs: aging, hippocampus, rat, theta rhythm.

Both memory and cognitive function decline with age in animals and humans. The limbic system, especially the hippocampus plays an important role in these functions. Therefore age-related impairment of these functions might be due at least in part to alterations in the hippocampus. The morphological and neurochemical age-related changes in rat hippocampus are well documented $[3,4,8,9,14,15$, $22,23,30]$, but fewer studies have examined the age-related changes in electrophysiological properties $[13,15,16,19$, 21].

Electroencephalograms (EEG) recorded from the rat hippocampus are highly correlated with functions such as memory and learning. Rat hippocampal EEGs are easily distinguished into two types. One is the theta rhythm (or rhythmic slow activity, RSA) and the other is the large amplitude irregular activity (LIA) $[6,24,25]$. The theta rhythm in particular is considered to be closely linked with hippocampal functions [29]. The theta rhythm can be further subdivided into two types. The theta rhythm observed in rat hippocampus with large movement is called type 1 theta. Type 2 theta is observed in REM sleep and anesthetized rats [6]. The type 2 theta can be abolished by acetylcholine antagonists (scopolamine or atropine) but these agents have no effect on type 1 theta $[6,25]$. Therefore, while the type 2 theta is generated by the cholinergic system, the type 1 theta is likely to arise from complex neurotransmitter systems $[6,27]$. The type 1 theta requires a connection to the isocortex via the entorhinal cortex, rather than depending on a particular neurotransmitter system [26]. A few reports have described changes in rat hippocampal theta with aging $[13,16]$, however, the correlation between the two types of

* Correspondence to: Toyosawa, K., Laboratory of Applied Physiology, Faculty of Agriculture, Kobe University, Rokkodai, Nada-ku, Kobe 657-0013, Japan. theta rhythms and aging remains unclear.

In this study, we attempted to clarify the age-related changes in the two types of hippocampal theta rhythms associated with different pharmacological systems.

\section{MATERIALS AND METHODS}

Male Wistar rats supplied by SLC Co., Ltd. were used in this study. For the measurement of the type 1 theta, three aged rats (22-25 months-old), four mature rats (12-13 months-old) and four young rats (3-4 months-old) were used. For the type 2 theta, four aged rats, four mature rats and three young rats were used. Aged and mature rats were purchased as retired rats (6-7 months-old) and housed in our animal facility for 6 months and 18 months respectively. The animal facility was maintained on a $12 \mathrm{hr}$ light/dark cycle, at $21 \pm 1{ }^{\circ} \mathrm{C}$ and $50-60 \%$ humidity.

The rats used for the type 1 theta experiments were deeply anesthetized by pentobarbital sodium $(50 \mathrm{mg} / \mathrm{kg}$, i.p. $)$ and then placed into a stereotaxic frame. A pair of staggered electrodes (200 $\mu \mathrm{m}$ stainless steel wires insulated except at cut ends) were chronically implanted in the bilateral hippocampal formation. The deep electrode was directed at the molecular layer of the dentate gyrus (DG), and the surface electrode at the CA1 pyramidal cell layer. Two stainless steel screw electrodes for reference and ground were fixed in the skull above the cerebellum. After 1 week of recovery from surgery, the type 1 theta recordings were carried out. The rats were placed in an open field box (100 $\times 70 \times 33 \mathrm{~cm}$ ) without a roof, which was painted gray. The hippocampal EEG and the behavioral data in the freely moving rats were recorded and stored simultaneously on a video tape using a video converter system (NEC Sanei) which received the signals from a multichannel amplifier and a CCD camera. Walking was selected as a type 1 theta 
related behavior because it is a simple behavior to distinguish from LIA related behavior, such as immobility. The type 1 theta recordings from the walking rats were sampled and analyzed (Fig. 1A).

In the type 2 theta experiment, rats were deeply anesthetized with urethane (1 g/kg, i.p.) and placed into a stereotaxic frame. Four wire electrodes for recording and two screw electrodes for reference and ground were set at the same coordinates as in the type 1 theta experiment. In addition, bipolar stimulating electrodes were implanted into the reticular pontin oralis nucleus (PON) for stable type 2 theta recording. The stimulating electrodes were cut so that one tip was $0.5 \mathrm{~mm}$ shorter than the other. The type 2 theta recording was started 60 min after urethane administration and performed for $2 \mathrm{hr}$. Stable type 2 theta rhythm was induced by the PON stimulation method as described by Vertes [28]. EEGs were stored on a PCM recorder (TEAC). After the theta rhythm recording from anesthetized rats, atropine was administered $(50 \mathrm{mg} / \mathrm{kg}$, i.p.) to the rats to confirm that the recorded theta rhythms were cholinergic (Fig. 1B).

All hippocampal EEGs were recorded monopolarly using one screw electrode as a reference. The other screw electrode was used as a ground. The electrodes were linked

\section{A. Type 1 Theta Rhythm (Free moving)}

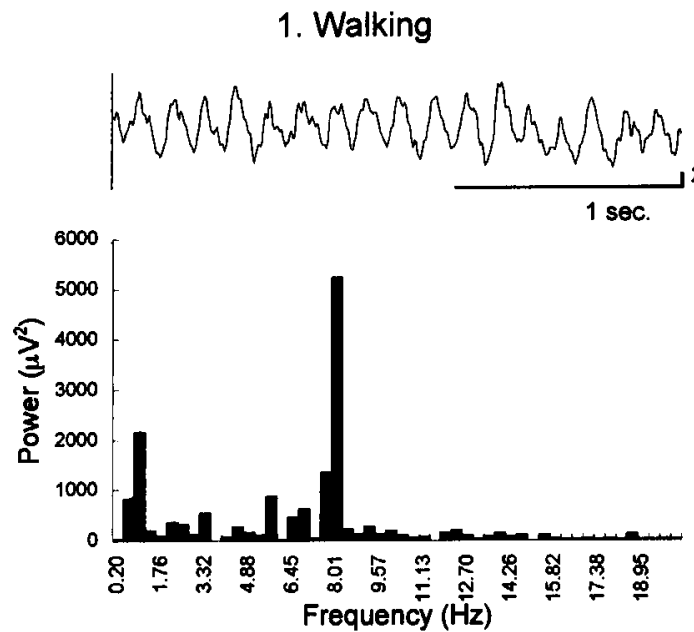

2. Immobility

$200 \mu \mathrm{V}$
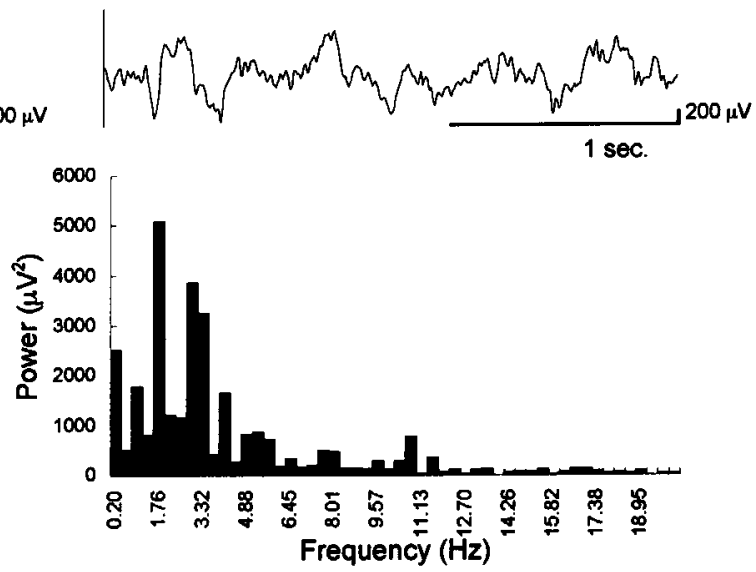

B. Type 2 Theta Rhythm (Urethane anesthetized)

1. After PON Stimulation

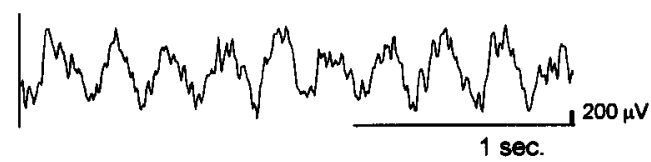

2. + Atropine $(50 \mathrm{mg} / \mathrm{kg}$, i.p. $)$

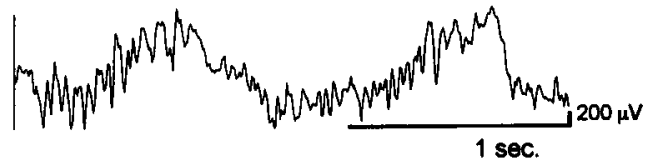

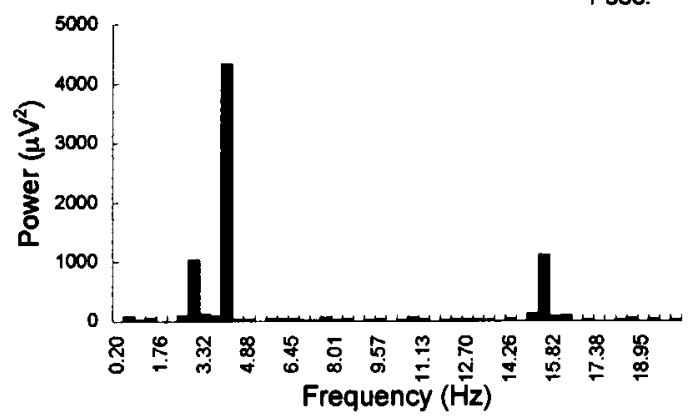

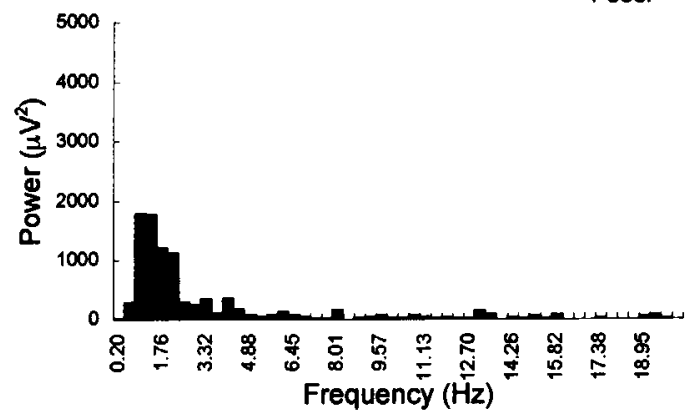

Fig. 1. The hippocampal EEG patterns of the theta rhythm and non-theta EEG and the power spectra calculated with FFT on each EEG. A: EEG recorded from the freely moving rat hippocampus (Type 1 theta recording condition), the type 1 theta recorded from the walking rat (1) and LIA recorded from the immobile rat (2). B: EEG from the urethane anesthetized rat hippocampus (the type 2 theta recording condition), the type 2 theta induced by PON stimulation (1) and abolishment of the type 2 theta by atropine administration (2). 
to a multichannel amplifier through flexible cables. The band pass filter was set from 0.5 to $100 \mathrm{~Hz}$.

After the EEG recordings, frozen brain sections $(40 \mu \mathrm{m})$ were made and stained with Cresyl Violet and the positions of the recording and stimulating electrodes were confirmed in all rats.

Both types of theta rhythm were analyzed off-line. Fifteen artifact-free theta lasting for at least 3 seconds of each type were selected from the tape recorded EEG (Fig. 2A, 2B). The selected EEG were digitized using a micro computer with an A/D converter, at $200 \mathrm{~Hz}$, for 2.56 seconds (512 points). Fast Fourier transformation was performed on each sample and the power spectrum was calculated.

The frequency indicating the largest power in the frequency band of each theta rhythm was found for all the power spectra, and evaluated as the peak frequency. The frequency bands of the type 1 theta and the type 2 theta were set to $6-12 \mathrm{~Hz}$ and $1.8-6 \mathrm{~Hz}$, respectively. The average of peak frequencies obtained from fifteen samples in one rat was considered as the individual data of the peak frequency in each theta rhythm. The power at the peak frequencies was calculated and evaluated as the peak power, in the same manner as the peak frequencies. The statistical differences in both of the peak frequency and the peak power among the young, the mature and the aged rats were

\section{A. Type 1 Theta Rhythm}

\section{Left CA1}

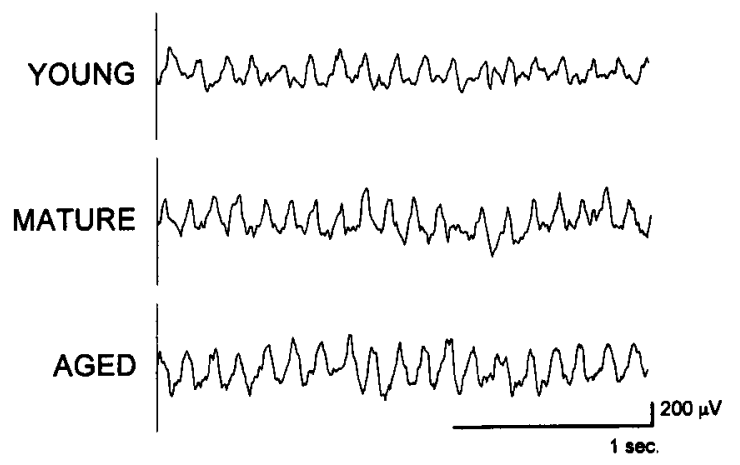

Left DG

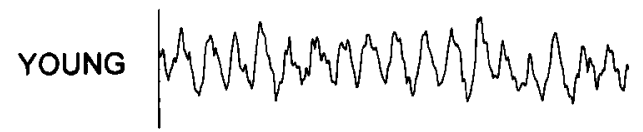

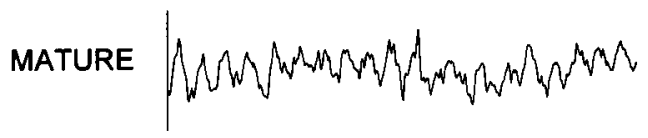

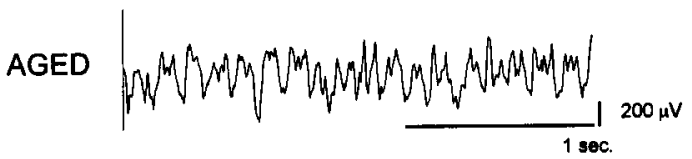

analyzed with one way analysis of variance (ANOVA).

\section{RESULTS}

The behavioral correlation of the type 1 theta described by Vanderwolf $[24,25]$ were well maintained in each age group and a peak was clearly observed in all power spectra (Fig. 1A). The peak frequency of the type 1 theta was observed between $7.0-8.3 \mathrm{~Hz}$ in all rats. The mean peak frequencies of the type 1 theta recorded from the young, mature and aged rats were $7.38 \pm 0.19 \mathrm{~Hz}, 7.48 \pm 0.10 \mathrm{~Hz}$, and $7.57 \pm 0.39 \mathrm{~Hz}$, respectively (mean $\pm \mathrm{SD}$, in left CA1). There were no significant differences among the three age groups, though the peak frequency of type 1 theta tended to shift to a higher range with aging (Fig. 3A). For the peak power of type 1 theta, the young rats showed the highest value and the mature and aged rats showed a lower value than the young rats. These decrements of peak power in the mature and aged rats were observed clearly in DG but not observed in CA1 (Table 1).

In type 2 theta rhythms, the peak frequencies of the young, mature, and aged rats were $3.98 \pm 0.29 \mathrm{~Hz}, 3.94 \pm$ $0.37 \mathrm{~Hz}$ and $2.20 \pm 0.30 \mathrm{~Hz}$, respectively (mean $\pm \mathrm{SD}$, in left CA1). The peak frequency of type 2 theta in the aged rats was significantly lower than those in the young and

\section{B. Type 2 Theta Rhythm}

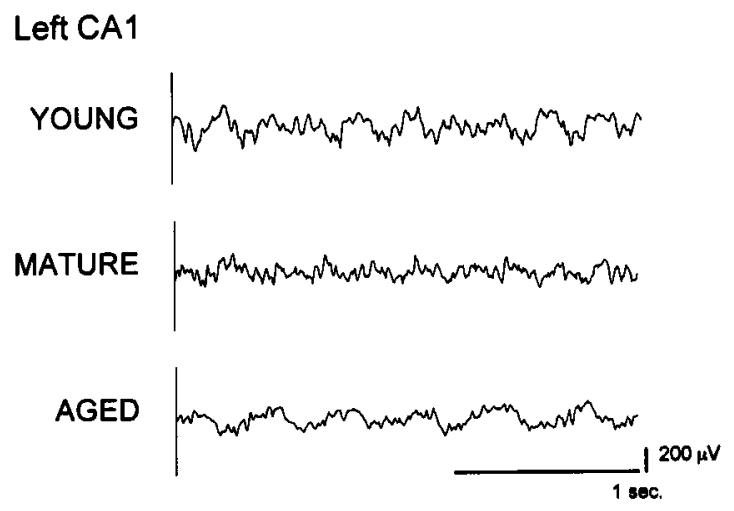

\section{Left DG}

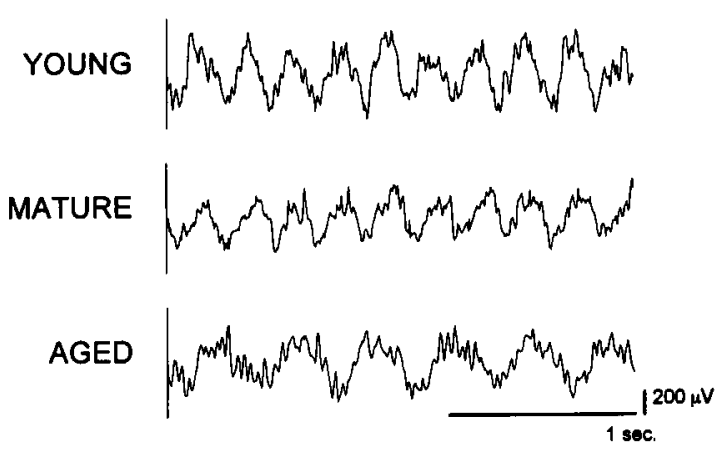

Fig. 2. Hippocampal theta rhythm recorded from the young, mature and aged rats. EEG recorded from the left hippocampus (the CA1 and the DG) are shown in this figure. A: type 1 theta rhythm, B: type 2 theta rhythm. 
Table 1. The peak power of the type 1 and the type 2 theta recorded from bilateral CA1 and DG in young, mature and aged rats

\begin{tabular}{cccc}
\hline & Young & Matured & Aged \\
\hline Type 1 theta & & & \\
Age (month) & 4 & 13 & $24-25$ \\
$\mathrm{n}$ & 4 & 4 & 3 \\
Peak power $\left(\mu \mathrm{V}^{2}\right) \pm \mathrm{SD}$ & & & \\
Left CA1 & $6299.48 \pm 7997.95$ & $6018.74 \pm 1671.92$ & $5421.03 \pm 4058.74$ \\
Right CA1 & $2721.75 \pm 3410.06$ & $3695.21 \pm 1718.24$ & $3582.77 \pm 2966.75$ \\
Left DG & $8884.57 \pm 4499.86$ & $4387.26 \pm 1204.19$ & $5264.45 \pm 1280.68$ \\
Right DG & $8177.10 \pm 6843.41$ & $4269.89 \pm 2215.47$ & $3897.34 \pm 2171.10$ \\
\hline Type 2 theta & & & \\
Age (month) & 3 & 4 & $22-25$ \\
$\mathrm{n}$ & 3 & & 4 \\
Peak power $\left(\mu \mathrm{V}^{2}\right) \pm \mathrm{SD}$ & & & \\
Left CA1 & $2650.00 \pm 1874.33$ & $3182.40 \pm 1426.17$ & $5115.65 \pm 4053.09$ \\
Right CA1 & $5086.31 \pm 2333.42$ & $1788.20 \pm 1073.03$ & $3815.65 \pm 3098.79$ \\
Left DG & $32798.88 \pm 24775.12$ & $10980.21 \pm 4445.86$ & $8809.85 \pm 7643.05$ \\
Right DG & $35251.01 \pm 12761.30$ & $6819.02 \pm 6040.30^{\text {a) }}$ & $9435.21 \pm 6725.49^{\text {a) }}$ \\
\hline Tight
\end{tabular}

The peak power in bilateral DG of mature and aged rats tended to decrease compared with young rats for both types of theta rhythm.

a) Significant differences were observed in the right DG of mature and aged rats compared with young rats $(\mathrm{p}<0.01)$.
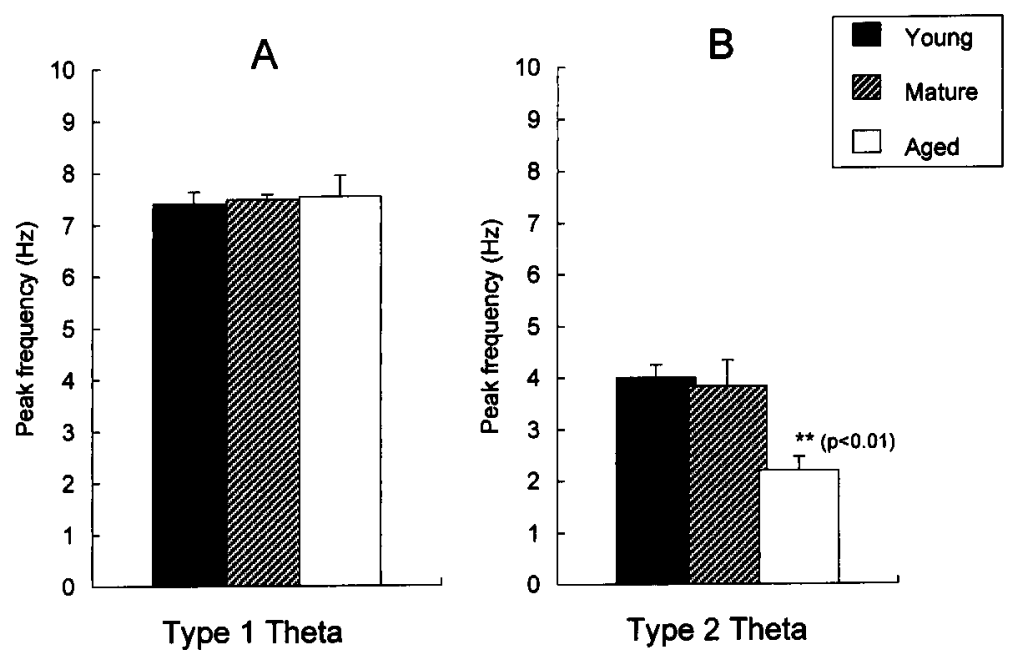

Fig. 3. The peak frequencies of the type 1 and the type 2 theta recorded from the left CA1 in the young, mature and aged rats. A: The peak frequencies of the type 1 theta recorded from the walking rats. B: The peak frequencies of the type 2 theta recorded from the anesthetized rats. In each age group, there were no differences among recording area of bilateral CA1 and DG. The peak frequencies of the type 1 theta do not change with age. On the other hand, the peak frequency of the type 2 theta in the aged rats significantly shifts to a lower range $(p<0.01)$ compared with the young and the matured rats. Bars in these figures represent SD.

mature rats $(\mathrm{p}<0.01)$ (Fig. 3B). The peak frequency of the type 2 theta, especially the $2.02 \mathrm{~Hz}$ in the aged rats, was out of the formal theta band, however, the PON-induced EEG recorded from the anesthetized rats maintained the thetalike rhythm clearly (Fig. 2B), and it was abolished by atropine application (Fig 1B). There was no significant difference in the peak frequency between the young and the mature rats.

The changes in the peak power of the type 2 theta were similar to those of the type 1 theta. The peak power in the mature and aged rats was lower than that in the young rats, especially in the DG. Though, a significant difference 
$(\mathrm{p}<0.01)$ was observed only in the right DG between the young and mature, and the young and aged rats. In the CA1 area, no age-related changes in peak power were observed (Table 1).

\section{DISCUSSION}

This study had two main findings. The peak power of both types of theta rhythm tended to decrease in the mature and aged rats in the DG compared with the young rats, and the peak frequency of the type 2 theta was decreased in the aged rats.

Several previous reports have described the changes in hippocampal theta rhythms with aging in rats. Lamour et $a l$. reported that the frequency of type 2 theta shifted to a lower range in aged rats [13]. Markowska et al. also reported that the peak frequency of the theta rhythms shifted to a lower range in aged rats [16]. They recorded the hippocampal theta from free-moving conditioned rats and the theta rhythms were still apparent after atropine application. Thus a shift in peak frequency in aged rats have been reported for both types of theta rhythms. In contrast to our findings, neither report observed any changes in peak power with age.

In the present study, a peak shift was only observed in the cholinergic type 2 theta rhythm. It is well known that the loss of basal forebrain cholinergic neurons is one of the most consistent and striking neuropathological features of Alzheimer's disease $[8,10]$. Therefore it was expected that age-related changes would be more clearly observed in the cholinergic system including the hippocampus.

The medial septal nucleus and the vertical limb of the diagonal band of Broca (MSN-VDB) are known to be a principal source of cholinergic input to the hippocampal formation [1]. Lamour et al. investigated hippocampal theta and firing of septal neurons [13]. They suggested that the decrease of neuronal firing rate in the septum was one reason for peak shifting of the theta in their report. However, the MSN-VDB area is well known as the pacemaker of hippocampal theta rhythms not only for type 2 but also type 1 theta $[6,7,20]$. Lesions of these areas caused the abolishment of all types of theta [7, 20]. Therefore the findings of this study suggest the possibility that the components for generating hippocampal theta other than the cholinergic system were still maintained in aged rats. Moreover, at least for the pacemaker of theta, the septohippocampal system (MSN-VDB-hippocampus) in aged rats has the ability to generate high frequency type 1 theta. The discrepancy of shifting of peak frequencies between type 1 walking and type 2 urethane anesthetized theta might be caused by the deterioration of the cholinergic system including not only the septo-hippocampal system but also other regions such as the brain stem, the region stimulated in this study. In addition, though the mechanism of the control of the theta rhythm other than the MSN-VDB remains unclear, these results suggest the possibility that the pacemaker in septo-hippocampal formation was compensated by the non-cholinergic neurotransmitter systems (the monoaminergic system might be included) [6, 27] or information processing between the isocortex and the hippocampus [26] under the type 1 theta condition, in the aged rat.

In this study, the peak power in the DG tended to decrease in the mature and aged rats, in both types of theta. It is known that the neurons called theta cells fire with the theta rhythms on phase in the CA1 and the DG where both types of theta are generated $[2,11,12]$. The theta cells at least in the CA1, are not pyramidal cells but rather interneurons $[11,12]$. The theta rhythms are generated not only by the theta cells but also other cells such as pyramidal cells and molecular cells [5]. The age-related decrease in neurons is more clearly observed in the CA1 [17]. Additionally the pyramidal cells in the CA1 are greatly damaged by ischemia [18]. It was expected that a decrease in the power of theta rhythms would be caused by a decrease in the number of concerned neurons; however, this hypothesis conflicts with histological findings. Moreover, the variance of peak power in the mature and aged rats was large, and some individuals in the mature and aged rats groups could generate the same power of theta as the young rats. Though the precise mechanism of the decrease in peak power of the theta rhythm remains unclear, it is likely that decreased peak power of the theta rhythm is a feature of age-related changes, at least in DG.

\section{REFERENCES}

1. Amaral, D. G. and Kurz, J. 1985. An analysis of the origins of the cholinergic and noncholinergic septal projections to the hippocampal formation of the rat. J. Comp. Neurol. 240: 37-59.

2. Alonso, A., Gaztelu, J. M., Buño, W. Jr. and García-Austt, E. 1987. Cross-correlation analysis of septohippocampal neurons during $\theta$ rhythm. Brain Res. 413: 135-146.

3. Biegon, A., Greenberger, V. and Segal, M. 1986. Quantitative histochemistry of brain acetylcholinesterase and learning rate in the aged rat. Neurobiol. Aging 7: 215-217.

4. Biegon, A., Duvdevani, R., Greenberger, V. and Segal, M. 1988. Aging and brain cholinergic muscarinic receptors: An autoradiographic study in the rat. J. Neurochem. 51: 13811385 .

5. Bland, B. H. and Whishaw, I. Q. 1976. Generators and topography of hippocampal theta (RSA) in the anaesthetized and freely moving rat. Brain Res. 118: 259-280.

6. Bland, B. H. 1986. The physiology and pharmacology of hippocampal formation theta rhythms. Prog. Neurobiol. 26: $1-54$.

7. Bland, S. K. and Bland, B. H. 1986. Medial septal modulation of hippocampal theta cell discharges. Brain Res. 375: 102-116.

8. Coleman, P. D. and Flood, D. G. 1987. Neuron numbers and dendritic extent in normal aging and Alzheimer's disease. Neurobiol. Aging 8: 521-545.

9. Consolo, S., Wang, J.-X., Fiorentini, F., Vezzani, A. and Ladinsky, H. 1986. In vivo and in vitro studies on the regulation of cholinergic neurotransmission in striatum, hippocampus and cortex of aged rats. Brain Res. 374: 212-218. 
10. Davies, P., Katzman, R. and Terry, R. D. 1980. Reduced somatostatine-like immunoreactivity in cerebral cortex from cases of Alzheimer disease and Alzheimer senile dementa. Nature (Lond.) 288: 279-280.

11. Fox, S. E. and Ranck, J. B. Jr. 1975. Localization and anatomical identification of theta and complex spike cells in dorsal hippocampal formation of rats. Exp. Neurol. 49: 299313.

12. Fox, S. E. and Ranck, J. B. Jr. 1981. Electrophysiological characteristics of hippocampal complex-spike cells and theta cells. Exp. Brain Res. 41: 399-410.

13. Lamour, Y., Bassant, M. H., Jobert, A. and Joly, M. 1989. Septo-hippocampal neurons in the aged rat: Relation between their electrophysiological and pharmacological properties and behavioral performances. Neurobiol. Aging 10: 181-186.

14. Lee, J. M., Ross, E. R., Gower, A., Paris, J. M., Martensson, R. and Lorens, S. A. 1994. Spatial learning deficits in the aged rat: Neuroanatomical and neurochemical correlates. Brain Res. Bull. 33: 489-500.

15. Lippa, A. S., Critchett, D. J., Ehlert, F., Yamamura, H. I., Enna, S. J. and Bartus, R. T. 1981. Age-related alterations in neurotransmitter receptors: An electrophysiological and biochemical analysis. Neurobiol. Aging 2: 3-8.

16. Markowska, A. L., Olton, D. S. and Givens, B. 1995: Cholinergic manipulations in the medial septal area: Age-related effects on working memory and hippocampal electrophysiology. J. Neurosci. 15: 2063-2073.

17. Meaney, M. J., O'Donnel, D., Rowe, W., Tannenbaum, B., Steverman, A., Walker, M., Nair, N.P.V. and Lupien, S. 1995. Individual differences in hypothalamic-pituitary-adrenal activity in later life and hippocampal aging. Exp. Gerontol. 30: 229-251.

18. Monmaur, P., Thomson, M. A. and M'harzi, M. 1986. Temporal changes in hippocampal theta activity following twenty minutes of forebrain ischemia in the chronic rat. Brain Res. 378: 262-273.

19. Potier, B., Rascol, O., Jazat, F., Lamour, Y. and Dutar, P. 1992. Alterations in the properties of hippocampal pyramidal neurons in the aged rat. Neuroscience 48: 793-806.

20. Rawlins, J. N. P., Feldon, J. and Gray, J. A. 1979. Septohippocampal connections and the hippocampal theta rhythm. Exp. Brain Res. 37: 49-63.

21. Segal, M. 1982. Changes in neurotransmitter actions in the aged rat hippocampus. Neurobiol. Aging 3: 121-124.

22. Sirviö, J., Pitkänen, A., Pääkköen, A., Partanen, J. and Riekkinen, P. J. 1989. Brain cholinergic enzymes and cortical EEG activity in young and old rats. Comp. Biochem. Physiol. 94C: $277-283$.

23. Valjakka, A., Sirviö, J., Pitkänen, A. and Riekkinen, P. J. 1990. Brain amines and neocortical EEG in young and aged rats. Comp. Biochem. Physiol. 96C: 299-304.

24. Vanderwolf, C. H. 1969. Hippocampal electrical activity and voluntary movement in the rat. Electroencephalogr. Clin. Neurophysiol. 26: 407-418.

25. Vanderwolf, C. H. 1975. Neocortical and hippocampal activation in relation to behavior: Effects of atropine, eserine, phenothiazines, and amphetamine. J. Comp. Physiol. Psychol. 88: 300-323.

26. Vanderwolf, C. H., Leung, L. W. and Cooley, R. K. 1985. Pathways through cingulate, neo- and entorhinal cortices mediate atropine-resistant hippocampal rhythmical slow activity. Brain Res. 347: 58-73.

27. Vanderwolf, C. H. and Baker, G. B. 1986. Evidence that serotonin mediates non-cholinergic low voltage fast activity, non-cholinergic hippocampal rhythmical slow activity and contributes to intelligent behavior. Brain Res. 374: 342-356.

28. Vertes, R. P. 1981. An analysis of ascending brain stem systems involved in hippocampal synchronization and desynchronization. J. Neurophysiol. 46: 1140-1159.

29. Winson, J. 1978. Loss of hippocampal theta rhythm results in spatial memory deficit in the rat. Science 201: 160-163.

30. Zoli, M., Ferraguti, F., Gustafsson, J. - $\AA$., Toffano, G., Fuxe, K. and Agnati, L. F. 1991. Selective reduction of glucocorticoid receptor immunoreactivity in the hippocampal formation and central amygdaloid nucleus of the aged rat. Brain Res. 545: 199-207. 